\title{
Two-degree-of-freedom fractional order-PID controllers design for fractional order processes with dead-time
}

\author{
Mingjie Li ${ }^{\mathrm{a}, *}$, Zhicheng Zhao ${ }^{\mathrm{b}}$, Jinggang Zhang ${ }^{\mathrm{b}}$, PingZhou $^{\mathrm{a}}$

\begin{abstract}
( ${ }^{a}$ State Key Laboratory of Synthetical Automation for Process Industries, Northeastern University, Shenyang 110819, China;
${ }^{\mathrm{b}}$ School of Electronic Information Engineering, Taiyuan University of Science and Technology, Taiyuan 030024, China)
\end{abstract}

\begin{abstract}
Recently, fractional order (FO) processes with dead-time have attracted more and more attention of many researchers in control field, but FO-PID controllers design techniques available for the FO processes with dead-time suffer from lack of direct systematic approaches. In this paper, a simple design and parameters tuning approach of two-degree-of-freedom (2-DOF) FO-PID controller based on internal model control (IMC) is proposed for FO processes with dead-time, conventional one-degree-of-freedom control exhibited shortcoming of coupling of robustness and dynamic response performance. 2-DOF control can overcome the above weakness which means it realizes decoupling of robustness and dynamic performance from each other. The adjustable parameter $\eta_{2}$ of FO-PID controller is directly related to the robustness of closed-loop system, and the analytical expression is given between the maximum sensitivity specification $M_{\mathrm{s}}$ and parameters $\eta_{2}$. In addition, according to the dynamic performance requirement of the practical system, the parameters $\eta_{1}$ could also be selected easily. By approximating the dead-time term of the process model with the first-order Padé or Taylor series, the expressions for 2-DOF FO-PID controller parameters are derived for three classes of FO processes with dead-time. Moreover, compared with other methods, this proposed method is simple and easy to implement. And finally, the simulation results are given to illustrate the effectiveness of this method.
\end{abstract}

Key words : 2-DOF FO-PID controller; FO processes with dead-time; IMC; maximum sensitivity; robustness tuning

\section{Introduction}

Nowadays, many natural and artificial systems have revealed fractional order inherent dynamic behavior, considerable attention has been paid to FO systems whose models are described by FO differential equations, though a few natural phenomena can be modeled or fitted in this way, FO model can provide a reliable modeling tool in describing many real dynamic processes. Many studies have shown that FO differential equation could provide a more accurate description of many real complex physical systems rather than conventional integer order equations [1-5]. With further research on fractional calculus theory in recent years, more and more mathematical models of practical systems have been gradually established by FO differential equation, such as heating-furnace [6], heat diffusion [7, 8], electrical circuits [9], gas turbine [10] and water distribution in a main irrigation canal pool [11] obtained by physical properties or system identification methods. However, in actual natural and engineering systems, dead-time is widespread and often affects the performance of the control system, making the stability of the control system reduce, or even causing instability of closed-loop system. Thus, research on control for the process with dead-time has more great theoretical and practical significance.

In recent years, since Podlubny I [12] proposed a generalization of the traditional PID controller based on fractional order calculus, namely $\mathrm{PI}^{\lambda} \mathrm{D}^{\mu}$ controller, and the integrator of order $\lambda$ and differentiator of order $\mu$ may assume real non-integer values, it has been shown the $\mathrm{PI}^{\lambda} \mathrm{D}^{\mu}$ controller has extra degrees-of-freedom introduced

\section{*Corresponding author.}

E-mail addresses: limingj88@126.com (Mingjie Li) by $\lambda$ and $\mu$, which can provide better performance of the system and robustness than classic integer order PID controller, so the design methods of FO-PID controller have attracted more and more attention in the control field. Various methods have been devised for designing and tuning of FO-PID controller. For example, papers [13-15] proposed a tuning method of FO-PD and FO-PI controllers respectively, these authors also have demonstrated better properties of these types of controllers than the classical integer order PID controllers, the method results in desired gain crossover frequency and phase margin, and then the phase Bode plot at the crossover frequency is flat, the tuning method can be used by means of solving three nonlinear equations. Ziegler-Nichols(Z-N) tuning rule has been developed for FO processes with dead-time [16]. A solution to the problem of stabilizing the given fractional order processes was by mapping into the global stability region of the parameters for FO-PID controllers in the space which were presented in [17-20]. The main contributions of $[21,22]$ were studies of design method in $\mathrm{FO}_{-} \mathrm{PD}^{\mu}$ and $\mathrm{FO}-\mathrm{PI}^{\lambda}$ controllers based on $H_{\infty}$ performance for FO processes with dead-time, and the stabilizing region of the parameters could be calculated via a range of frequencies respectively, which satisfied the $H_{\infty}$-norm constraint of complementary sensitivity function and defined the $H_{\infty}$ boundary curve. Meanwhile, many intelligent optimization tuning methods of FO-PID controllers based on improved reduced FO processes with dead-time were argued in [23-26], by optimizing the integrated absolute error criterion or minimizing time domain performance index of the control system that were given respectively. A design technique of the hybrid fuzzy FO-PID controller was investigated for FO process with dead-time by minimizing the integrated absolute criterion and deviation in control signal [27, 28]. In [29-31] a new approach was developed to design simple FO-PID controllers based on internal model control paradigm for FO processes. In many of these above studies, these focuses have been made to design and tune FO-PID controllers for FO processes directly or to 
improve reduced FO processes with dead-time by enhancing dynamic performance and robustness of the closed-loop control system.

In this paper, we focus on FO processes with dead-time controlled by 2-DOF FO-PID controllers, but a new approach will be investigated. 2-DOF FO-PID control structure based on the principle of IMC is proposed, which can make the robustness and dynamic performance of the system decoupled from each other, the adjustable parameter $\eta_{2}$ of the FO controller is directly related to the robustness of the closed-loop system, and the analytical expressions is given between $M_{\mathrm{s}}$ and the parameter $\eta_{2}$ of FO-PID controller. According to the dynamic performance requirement of practical system, parameters $\eta_{1}$ can also be selected easily. And different types of 2-DOF FO-PID controllers can be obtained for three classes of FO processes with dead-time by approximating the dead-time term of processes models with the first-order Padé or Taylor series. So, 2-DOF FO-PID controller parameters tuning can rely on the performance of the set-point tracking or robustness independently and monotonously, the shortcoming of the conventional one degree of freedom IMC could be avoided. The simulation results show that the proposed method is effective.

\section{Preliminary}

\subsection{Fractional order calculus}

Fractional calculus is a generalization of the integration and differentiation to the non-integer (fractional) order fundamental operator ${ }_{a} D_{t}^{\gamma} f(t)$. A commonly used definition of the FO calculus is Riemann-Liouville (RL) and Grünwald-Letnikov (GL) definition. If the function $f(t)$ is continuous and differentiable in the interval $[a, t]$. According to $\mathrm{RL}$ definition, the $\gamma$-th order integration of a function $f(t)$ can be expressed as

$$
{ }_{a}^{\mathrm{RL}} D_{t}^{\gamma} f(t)=\frac{1}{\Gamma(-\gamma)} \int_{a}^{t} \frac{f(\tau)}{(t-\tau)^{\gamma+1}} d \tau, \gamma<0
$$

and similarly the $\gamma$-th order differentiation of the function $f(t)$ is defined as

$$
{ }_{a}^{\mathrm{RL}} D_{t}^{\gamma} f(t)=\frac{1}{\Gamma(n-\gamma)} \frac{d^{n}}{d t^{n}} \int_{a}^{t} \frac{f(\tau)}{(t-\tau)^{\gamma-n+1}} d \tau, \gamma>0
$$

For $n-1<\gamma<n, \Gamma(\bullet)$ is the Euler's gamma function, $a$ and $t$ are the limits and $\gamma$ is the order of the operation.

And an alternative definition based on the concept of fractional differentiation is GL definition given by

$$
{ }_{a}^{\mathrm{GL}} D_{t}^{\gamma} f(t)=\lim _{h \rightarrow 0} \frac{1}{h^{\gamma}} \sum_{k=0}^{[(t-a) / h]}(-1)^{k}\left(\begin{array}{l}
\gamma \\
k
\end{array}\right) f(t-k h)
$$

where ['] means the integer part, $h$ represents sampling period and

$$
\left(\begin{array}{l}
\gamma \\
k
\end{array}\right)=\frac{\gamma(\gamma-1)(\gamma-2) \ldots . .(\gamma-k+1)}{k !}
$$

The Laplace transform form of a $\gamma$-th derivative operator with $\gamma \in \square_{+}$of a signal $f(t)$ is given by

$$
L\left\{{ }_{a} D_{t}^{\gamma} f(t)\right\}=s^{\gamma} L\{f(t)\}-\sum_{\mathrm{i}=0}^{k-1} s^{\gamma-1-i}{ }_{a}^{\gamma} f(0)
$$

where $k-1<\gamma<k$ and $k \in N$, which in zero initial conditions can be reduced to

$$
L\left\{{ }_{a} D_{t}^{\gamma} f(t)\right\}=s^{\gamma} F(s)
$$

In the present simulation study, to implement the FO operator $s^{\gamma}$, it is employed by oustaloup recursive algorithm [32] for the approximation. If the expected frequency range is selected as $\left[\omega_{a}, \omega_{b}\right]$, to acquire a good accuracy in this frequency range, the approximate transfer function of a continuous FO operators $s^{\gamma}$ via integer order components with oustaloup recursive algorithm is calculated as follows

$$
G_{f}(s)=s^{\gamma} \approx K \prod_{j=-N}^{N} \frac{s+w_{j}}{s+w_{j}^{\prime}}, \quad(0<\gamma<1)
$$

where the zeros, poles and gain $K$ can be evaluated respectively from

$$
\begin{aligned}
& \omega_{j}=\omega_{a}\left(\frac{\omega_{b}}{\omega_{a}}\right)^{\frac{K+N+0.5(1-\gamma)}{2 N+1}} \\
& \omega_{j}^{\prime}=\omega_{a}\left(\frac{\omega_{b}}{\omega_{a}}\right)^{\frac{K+N+0.5(1+\gamma)}{2 N+1}} \\
& K=\left(\frac{\omega_{b}}{\omega_{a}}\right)^{-\frac{\gamma}{2}} \prod_{j=-N}^{N} \frac{\omega_{j}^{\prime}}{\omega_{j}}
\end{aligned}
$$

where $\gamma$ is the order of the differ-integration and $(2 N+1)$ is order of the filter. In our simulation, for the approximation of FO differentiator, frequency range of practical interest is set to be from $0.001 \mathrm{~Hz}$ to $1000 \mathrm{~Hz}$, and $N$ is selected as 4 for the proper accuracy of the approximation. If $\gamma>1$, FO derivative can be written as $s^{\gamma}=s \cdot s^{\gamma-1}$, where only fractional part is approximated via Eq. (7).

\subsection{Fractional order PID controller}

The classical Proportional-Integral-Derivative (PID) controller is still widely recognized as one of the simplest yet most effective control strategies in the control industry. Based on the principles of fractional calculus, the following four types of FO-controllers have been briefly introduced in [33], namely, CRONE controller, FO-lead-lag compensateor, FO-TID (Tilt-Inte -gral-Derivateive) controller, and $\mathrm{PI}^{\lambda} \mathrm{D}^{\mu}$ controller. For example, fractional order TID controller, which has structure similar to a traditional integer order PID controller but the proportional component is replaced with a tilted component having a transfer function $s$ to the power of $(-1 / n)$. The resulting transfer function of TID controller has the form

$$
C(s)=\frac{T_{\mathrm{i}}}{s^{1 / n}}+\frac{K_{\mathrm{i}}}{s}+K_{\mathrm{d}} s,(n>0)
$$

where $T_{\mathrm{i}}, K_{\mathrm{i}}$, and $K_{\mathrm{d}}$ are the controller constants, and $n$ is a non-zero real number, preferably $n \in(2,3)$.

And replacing the Laplace variable $s$ by fractional powers of $s$, the fractional-order controller $\mathrm{PI}^{\lambda} \mathrm{D}^{\mu}$ was defined as a generalization of the traditional integer order PID controller with integrator of real order $\lambda$ and differentiator of real order $\mu$ by following transfer function 


$$
C(s)=K_{\mathrm{p}}+\frac{K_{\mathrm{i}}}{s^{\lambda}}+K_{\mathrm{d}} s^{\mu},(\lambda, \mu>0)
$$

where $K_{\mathrm{p}}$ is proportional constant, $K_{\mathrm{i}}$ is integration constant, $K_{\mathrm{d}}$ is differentiation constant.

Similarly, another two different structures of FO-PID controllers with a filter was [26, 34] defined, whose transfer functions are given as follows, respectively.

$$
\begin{aligned}
& C(s)=K_{\mathrm{p}}\left(\frac{T_{i} s^{\lambda}+1}{T_{i} s^{\lambda}}\right)\left(\frac{T_{\mathrm{d}} s^{\mu}+1}{1+T_{\mathrm{d}} s / N}\right) \\
& C(s)=K_{\mathrm{p}}\left(1+\frac{1}{T_{i} s}\right)^{\lambda}\left(\frac{T_{\mathrm{d}} s+1}{1+T_{\mathrm{d}} s / N}\right)^{\mu}
\end{aligned}
$$

FO-PD and FO-PI controllers were proposed and designed for different processes [13-15] based on Eq.(11) and (12).

The FO-PID controller can be put in the form of FO-PI ${ }^{\lambda} D^{\mu}$ structure cascaded with a fractional filter in [31], the general structure of the FO controller is given by

$$
C(s)=H(s) \cdot K_{\mathrm{p}}\left(1+\frac{1}{T_{i} s^{\lambda}}+T_{\mathrm{d}} s^{\mu}\right)
$$

where $H(s)$ is a fractional filter.

It has also been found that a simple form of FO-I ${ }^{\lambda} \mathrm{D}^{\mu}$ controller was presented in $[36,37]$. On the other hand, the FO-PDD ${ }^{0.5}$ control scheme was discussed for double integral plant [38]. Meanwhile, authors also have demonstrated that these different types of FO-PID controllers were not only more flexible in the controller design, but also improved dynamic performance of the closed-loop systems and had better robustness than traditional integer order PID controllers.

\section{2-DOF FO-PID controller design}

\subsection{2-DOF control based on IMC}

IMC is a well known advanced control strategy which capable of handling control process perfectly and has been applied in industrial process. Unfortunately, IMC controller not only provides slow response but also displays coupling of robustness and dynamic response performance. In order to overcome these weaknesses, 2-DOF control strategy based on IMC is developed. The basic structure of 2-DOF based on IMC is shown in Fig.1, where $G(s)$ is the process, $M(s)$ is the model of the process, $Q_{1}(s)$ and $Q_{2}(s)$ is 2-DOF internal model controller respectively. The $d(s), r(s)$ and $y(s)$ correspond to the disturbance, the input and output of the system, respectively.

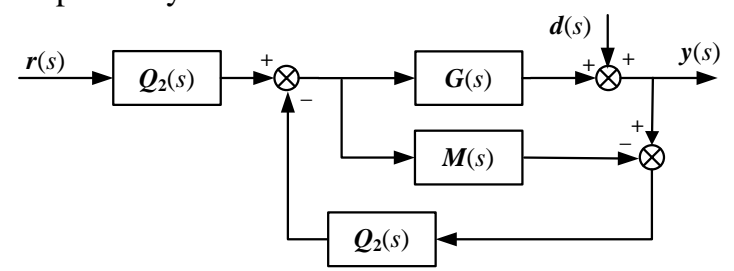

Fig.1 Structure of 2-DOF control based on IMC

The 2-DOF based on IMC structure is shown in Fig.1, which can be reduced to the equivalent structure shown in Fig.2, where $C(s)$ is the 2-DOF controller, $F(s)$ is the set-point filter.

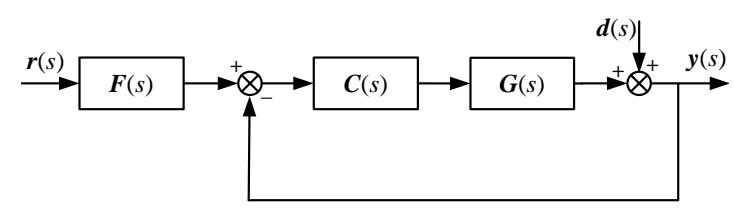

Fig.2 Equivalent structure of 2-DOF control

It can be obtained by Fig. 2:

$$
y(s)=G_{r}(s) r(s)+G_{d}(s) d(s)
$$

where

$$
\begin{aligned}
& G_{r}(s)=\frac{F(s) C(s) G(s)}{1+C(s) G(s)} \\
& G_{d}(s)=\frac{1}{1+C(s) G(s)}
\end{aligned}
$$

It can also be obtained by Fig. 1 and Fig. 2

$$
\begin{aligned}
y(s)= & \frac{G(s) Q_{1}(s)}{1+Q_{2}(s)[G(s)-M(s)]} r(s)+ \\
& \frac{1-M(s) Q_{2}(s)}{1+Q_{2}(s)[G(s)-M(s)]} d(s)
\end{aligned}
$$

The 2-DOF controller $C(s)$ and set-point filter $F(s)$ via Eq.(10) (11) and (12) respectively are

$$
C(s)=\frac{Q_{2}(s)}{1-M(s) Q_{2}(s)}, F(s)=\frac{Q_{1}(s)}{Q_{2}(s)}
$$

Based on Eq. (18), $C(s)$ and $F(s)$ can be obtained by $Q_{1}(s)$ and $Q_{2}(s)$. If there is no error in the model, namely $G(s)=M(s)$, Eq. (17) can be simplified to

$$
Y(s)=M(s) Q_{1}(s) r(s)+\left[1-M(s) Q_{2}(s)\right] d(s)
$$

It's well known by Eq.(19) that $Q_{1}(s)$ may change the speed of dynamic response of the system, meanwhile the sensitivity function and disturbance rejection property of the control system is consistent with Eq.(17), so $Q_{2}(s)$ also directly affects disturbance rejection property and robustness of the closed-loop control system.

In this paper, three different types of the FO processes with dead-time are considered with the following transfer function

$$
\begin{aligned}
G_{1}(s) & =\frac{K}{T s^{\alpha}+1} e^{-L s} \\
G_{2}(s) & =\frac{K}{T_{1} s^{2 \alpha}+T_{2} s^{\alpha}+1} e^{-L s} \\
G_{3}(s) & =\frac{K}{s\left(T s^{\alpha}+1\right)} e^{-L s}
\end{aligned}
$$

where $K, T, T_{1}, T_{2}$ and $L$ are positive real number. In this paper, we considered $\alpha \in(0,2)$ in the above process models.

According to the design procedure for IMC system, the process model is decomposed into two parts:

$$
M(s)=M_{+}(s) M_{-}(s)
$$

where $M_{+}(s)$ that contains all the dead-time and right-half plane zeros of $M(s)$ is the singular part of $M(s)$. Thus, the steady-state gain of $M(s)$ will remain in $M(s)$, which is used to design the IMC controller. And $M_{-}(s)$ is the 
nonsingular part. Therefore, internal model controller $Q_{1}(s)$ and $Q_{2}(s)$ can be specified as

$$
Q_{1}(s)=\frac{1}{M_{-}(s)} f_{1}(s), Q_{2}(s)=\frac{1}{M_{-}(s)} f_{2}(s)
$$

where $f_{1,2}(s)$ is a low-pass filter and is usually chosen as follows respectively

$$
f_{1}(s)=\frac{1}{\left(1+\eta_{1} s\right)^{r}}, f_{2}(s)=\frac{1}{\left(1+\eta_{2} s\right)^{r}}
$$

Parameter $r$ is a positive integer choice so that the controller $C(s)$ is realizable. Also, $\eta_{1}$ and $\eta_{2}$ are the filter time constant, determined by the expected system performance. The $f_{i}(s)(i=1,2)$ is a low pass filter with a steady-state gain of one. So, a typical form of low-pass filter $f_{1}(s)=1 /\left(1+\eta_{1} s\right)$ and $f_{2}(s)=1 /\left(1+\eta_{2} s\right)$ are selected respectively, the controller $Q_{1}(s)$ and $Q_{2}(s)$ can be obtained for FO process with dead-time by IMC principle

$$
\begin{aligned}
& Q_{1}(s)=M_{-}^{-1}(s) f_{1}(s)=\frac{M_{-}^{-1}(s)}{\left(1+\eta_{1} s\right)} \\
& Q_{2}(s)=M_{-}^{-1}(s) f_{2}(s)=\frac{M_{-}^{-1}(s)}{\left(1+\eta_{2} s\right)}
\end{aligned}
$$

So, the equivalent feedback 2-DOF controller $C(s)$ and set-point filter $F(s)$ can be calculated as follows respectively based on Eq.(20) (21) and (22)

$$
\begin{aligned}
& C_{1}(s)=\frac{T s^{\alpha}+1}{K\left(1+\eta_{2} s-e^{-L s}\right)} \\
& C_{2}(s)=\frac{T_{1} s^{2 \alpha}+T_{2} s^{\alpha}+1}{K\left(1+\eta_{2} s-e^{-L s}\right)} \\
& C_{3}(s)=\frac{\left(T s^{\alpha}+1\right) s}{K\left(1+\eta_{2} s-e^{-L s}\right)} \\
& F(s)=\frac{1+\eta_{1} s}{1+\eta_{2} s}
\end{aligned}
$$

It's obviously observed that $C_{i}(s)(i=1,2,3)$ and $F(s)$ have only two adjustable parameters $\eta_{1}$ and $\eta_{2}$, the performance of set-point tracking and robustness can be adjusted by $\eta_{1}$ and $\eta_{2}$ separately and monotonously. It is clear for the 2-DOF control scheme that two parameters need to be tuned, $\eta_{2}$ is responsible for the robustness of the closed-loop control system, and $\eta_{1}$ is solely for the performance of set-point tracking.

\subsection{Tuning of 2-DOF FO controller}

Robustness is always of primary concern for process control when the control systems are designed and analyzed because the models used for the design of controllers are usually imprecise and the parameters of all physical systems vary with the working condition and time. So it is important that the controller parameters are chosen in such a way that the closed-loop system is not too sensitive to the modeling error and variations in process dynamics. It was pointed out by Astrom et al [39] that sensitivity to model errors can be expressed as the largest value of the sensitivity function. As an effective robustness measure, the maximum sensitivity $\left(M_{s}\right)$ is defined as follows

$$
M_{s}=\max _{\omega \rightarrow+\infty}\left|\frac{1}{1+C(j \omega) G(j \omega)}\right|
$$

The geometric interpretation of the maximum sensitivity is shown in Fig.3.The quantity $M_{\mathrm{s}}$ is also the inverse of the shortest distance from the Nyquist curve of the open-loop transfer function $L(j \omega)=C(j \omega) G(j \omega)$ to the critical point $(-1, j 0)$ in the complex plane. What is called the $M_{s}$ circle is a circle drawn with -1 center and $1 / M_{s}$ radius. Typical values of $M_{s}$ are in the range of 1.2-2.0. In other words, the Nyquist curve of the open-loop transfer function is tangent to a circle around the critical point with radius $1 / M_{s}$.

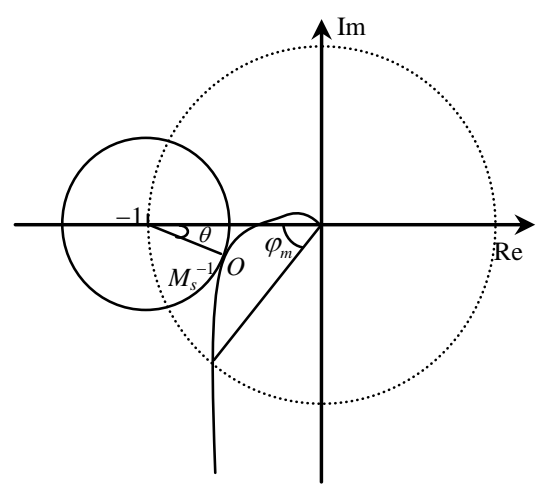

Fig.3 Geometric illustration of maximum sensitivity

Obviously, the higher the value of $M_{\mathrm{s}}$ is, the less robust the system is to modelling uncertainties. In fact, as $M_{\mathrm{s}}$ increasing the Nyquist curve approaches to the critical point, the Nyquist curve intersects the critical point. Instead of maximizing the sensitivity function with respect to frequency, the sensitivity function is bounded for all frequencies, not just at a particular frequency. Gain and phase margins specifications are two well-known measures of robustness, $M_{s}$ measures the closeness of the Nyquist curve from the critical point at all frequencies and not just the two frequencies as associated with gain and phase margins, so it can serve as a better measure of system robustness. The sensitivity is related to the gain and phase margin conditions [40, 41], which guarantees the following margins.

$$
\begin{gathered}
h>\frac{M_{s}}{M_{s}-1} \\
\varphi_{m}>2 \arcsin \frac{1}{2 M_{s}}
\end{gathered}
$$

where $h$ and $\varphi_{m}$ is the gain and phase margin, respectively. $\theta$ is the angle that between the negative real axis and the line drawn connecting the critical point and the point on the Nyquist curve.

In Fig3, assuming that point $O$ is the tangent point. The condition that the Nyquist curve of open-loop transfer function $L(j \omega)$ goes through point $O$. Thus, the maximum sensitivity can satisfy both gain and phase margin specifications. In order to use the sensitivity specification in the design of the controller, the condition that the open-loop transfer function $L(j \omega)=C(j \omega) G(j \omega)$ goes through the point $O$ on the Nyquist curve is 


$$
\left\{\begin{array}{l}
L(j \omega)=-1+\frac{1}{M_{s}} e^{-j \varphi} \\
\arg \frac{d L(j \omega)}{d \omega}=\frac{\pi}{2}-\varphi
\end{array}\right.
$$

From Eq.(20) and (28), Eq.(21) and (29) or Eq.(22) and (30), the open-loop transfer function of the system can be obtained

$$
L(s)=\frac{e^{-L s}}{1+\eta_{2} s-e^{-L s}}=\frac{1}{\left(1+\eta_{2} s\right) e^{L s}-1}
$$

Then $s=j \omega$ is brought into the above Eq.(36) in which $e^{j \omega L}=\cos (\omega L)+j \sin (\omega L)$. Noting that,

$$
\begin{aligned}
L(j \omega) & =\frac{1}{\left(1+j \eta_{2} \omega\right) e^{j \omega L}-1}=\frac{1}{D_{r}(\omega)+j D_{i}(\omega)} \\
& =\frac{D_{r}(\omega)-j D_{i}(\omega)}{\sqrt{D_{r}^{2}(\omega)+D_{i}^{2}(\omega)}}
\end{aligned}
$$

where

$$
\left\{\begin{array}{l}
D_{r}(\omega)=\cos (\omega L)-\eta_{2} \omega \sin (\omega L)-1 \\
D_{i}(\omega)=\eta_{2} \omega \cos (\omega L)+\sin (\omega L)
\end{array}\right.
$$

Based on the above Eq. (35) and (38), assuming that $a=\omega L$, we can obtain the following:

$$
\left\{\begin{array}{l}
D_{r}(a)=\cos (a)-a \eta_{2} / L \sin (a)-1 \\
D_{i}(a)=a \eta_{2} / L \cos (a)+\sin (a)
\end{array}\right.
$$

The Eq. (40) can be obtained coming from Eq. (35) and (39):

$$
\left\{\begin{array}{l}
\frac{D_{r}(a)}{\sqrt{D_{r}^{2}(a)+D_{i}^{2}(a)}}=-1+\frac{1}{M_{s}} \cos (\varphi) \\
\frac{D_{i}(a)}{\sqrt{D_{r}^{2}(a)+D_{i}^{2}(a)}}=\frac{1}{M_{s}} \sin (\varphi) \\
\arg \frac{d L(j a)}{d a}=\frac{\pi}{2}-\varphi
\end{array}\right.
$$

Equation (40) is a nonlinear equation and has no direct solution. However, if the value of $M_{s}$ is given in advance, as a result, $a, \eta_{2} / L$ and $\varphi$ are three unknown parameters in the above Eq.(40). Nonlinear equation (40) can be solved easily by Newton iterative method when $M_{\mathrm{s}}$ is given between 1.2 and 2.0 with step value of 0.05 respectively. So a set of values of $\eta_{2} / L$ and $M_{\mathrm{s}}$ can be obtained, and then curve fitting technique based on these data of $\eta_{2} / L$ and $M_{\mathrm{s}}$ is employed to explore this relationship between the adjustable parameter $\eta_{2} / L$ and $M_{s}$. The following approximating results of parameter $\eta_{2}$ are shown in Fig.4, the approximating relationship between the adjustable parameter $\eta_{2}$ and $M_{s}$ can be expressed by fitting the values of $\eta_{2} / L$ and $M_{s}$ as follows

$$
\eta_{2}=\left(\frac{12.07}{M_{s}^{7.12}}+0.425\right) L
$$

So, the expression makes it easy to find the adjustable parameter $\eta_{2}$ of the FO controller with a given $M_{s}$. Seeing from Fig.4, the value of $\eta_{2}$ decreases gradually along with the increasing of value of $M_{s}$, Meanwhile, in order to give a better speed of dynamic response of the system, $\eta_{1}$ is always smaller than $\eta_{2}$.

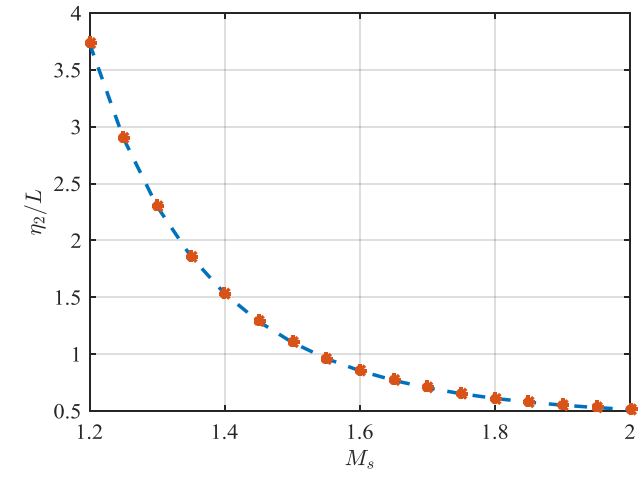

Fig.4 Relation between parameter $\eta_{2} / L$ and $M_{\mathrm{s}}$

\section{Simulation and results}

To demonstrate the effectiveness of the proposed controller design method, the closed-loop system performance is evaluated. Output performance $y(t)$ is quantified by computing the integral of time multiplied by absolute error $\left(J_{\mathrm{ITAE}}\right)$ criterion for both disturbance rejection and set-point tracking, the $J_{\mathrm{ITAE}}$ criterion is defined as

$$
J_{\mathrm{ITAE}}=\int_{0}^{\infty} t|r(t)-y(t)| d t
$$

Overshoot $(\sigma)$ is a measure of how much the response exceeds the ultimate value following a step change in set-point, and smaller values of these criterions imply better performance. Moreover, the achieved performance indexes can also be used for the assessment of the controller performance. The proposed method will be illustrated by the following three different types of FO processes with dead-time.

Example 1. In [16], a comparative study is done on frequency and time domain tuning strategies for FO-PID controllers to handle a FO process with dead-time. The process transfer function is given as:

$$
G_{1}(s)=\frac{K}{T s^{\alpha}+1} e^{-L s}=\frac{1}{s^{0.5}+1} e^{-0.5 s}
$$

In order to obtain the FO-PID controller, the dead-time term is approximated by a first-order Padé in Eq. (28), namely $e^{-L s} \approx(1-0.5 L s) /(1+0.5 L s)$, and the standard of 2-DOF FO-PID controller in Eq. (11) can be obtained as a generalization of conventional integer order PID controller form based on the proposed method

$$
C_{1}(s)=\frac{0.25}{\left(\eta_{2}+0.5\right)}\left(\frac{0.25 s+1}{0.25 s}\right)\left(\frac{s^{0.5}+1}{1+0.5 \eta_{2} s /\left(\eta_{2}+0.5\right)}\right)
$$

The maximum sensitivity $M_{\mathrm{s}}=1.7$ is chosen. Hence, the adjustable parameter $\eta_{2}=0.352$ of 2-DOF FO-PID controller can be tuned via Eq.(41), and the adjustable parameter $\eta_{1}=0.3$ and $\eta_{1}=0.2$ are selected respectively. In addition, the proposed method is compared with two other FO-PID [16]: well-known Ziegler-Nichols and single-degree-of-freedom IMC method, the FO-PID controllers obtained using the method proposed in [16] are given by

$$
\begin{gathered}
C(s)=1.4098+\frac{1.6486}{s^{1.1011}}-0.2139 s^{0.1855} \\
C(s)=0.5+\frac{2}{s}+0.5 s^{0.5}
\end{gathered}
$$


When the process model is accurate, the simulation results are shown for these three design methods, $r$ is a unit step applied at $t=0$ and a negative step disturbance of magnitude 0.2 is inserted at $t=15 \mathrm{~s}$ in Fig.5. The performance indices are listed in Tab.1.

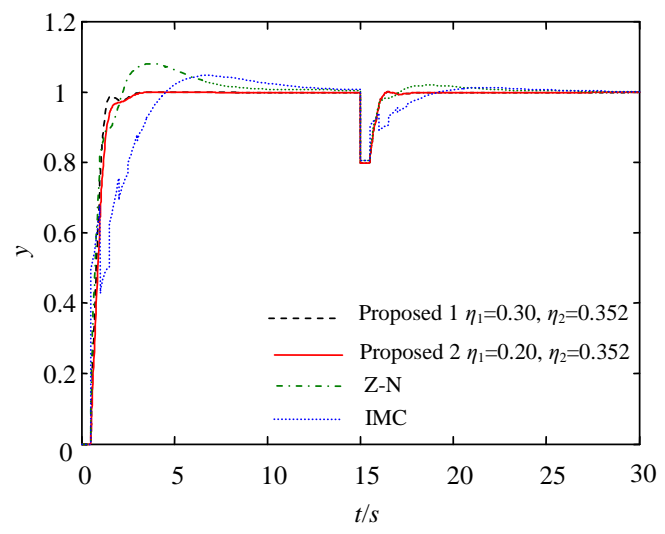

Fig.5 Step responses with nominal process for example 1

Now suppose that the process model generates perturbation Eq.(47), the robustness of the proposed method is evaluated. The simulation results for the model mismatch of the three methods are given in Fig.6 and Tab.1.

$$
G_{1}^{\prime \prime}(s)=\frac{0.9}{0.9 s^{0.5}+1} e^{-0.6 s}
$$

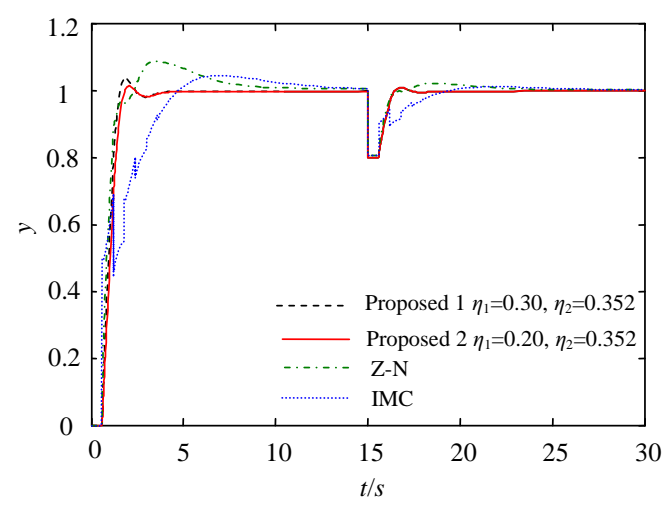

Fig.6 Step responses with perturbed process for example 1

The results of example $G_{1}$ clearly show that the proposed method gives both smaller overshoot and faster disturbance rejection while maintaining set-point performance in Fig.5 and Tab.1. The proposed method with minimum values of $J_{\text {ITAE }}$ and $\sigma$ clearly shows a better performance than the other two methods. The superior robustness of the proposed method was demonstrated by the smaller values of performance indices in Tab.1.

Example 2. The following class of fractional order process with dead-time given in [42] is considered

$$
G_{2}(s)=\frac{K}{T_{1} s^{2 \alpha}+T_{2} s^{\alpha}+1} e^{-L s}=\frac{1}{8 s^{1.5}+5 s^{0.75}+1} e^{-4.8 s}
$$

Based on the first order Taylor approximation of time-delay term, namely $e^{-L s} \approx 1-L s$, a standard 2-DOF FO-PID controller $C_{2}(s)$ can be calculated via Eq.(29) which is expressed

$$
C_{2}(s)=\frac{5}{\left(\eta_{2}+4.8\right) s^{0.25}}\left(1+\frac{0.2}{s^{0.75}}+1.6 s^{0.75}\right)
$$

The FO-PID controller can be put in the form of FO-PI ${ }^{\lambda} D^{\mu}$ structure cascaded with a fractional filter in [31], which is also defined as a generalization of conventional integer order PID controller.

In this paper, the integer order PID controller is tuned by reducing the order of example 2 as the listed first order model with dead-time

$$
G(s)=\frac{K}{T s+1} e^{-L s}=\frac{0.8639}{6.8178 s+1} e^{-5.5339 s}
$$

Then PID controller parameters could be achieved by classical Ziegler-Nichols method

$$
K_{\mathrm{p}}=\frac{1.2 T}{K L}, T_{\mathrm{i}}=2 L, T_{\mathrm{d}}=0.5 L
$$

Similarly, the maximum sensitivity $M_{\mathrm{s}}=1.5$ is chosen. Hence, the adjustable parameter $\eta_{2}=5.2698$ of controller can be tuned via Eq.(41). In addition, the parameter $\eta_{1}=4.0$ and $\eta_{1}=3.0$ is chosen respectively, the control performance of the proposed method is compared with Z-N tuning method. When the model is accurate, the closed-loop control system is simulated with a step increase of magnitude 1 at the reference input at $t=0$ and a step disturbance of magnitude 0.2 at the process output at $t=100 \mathrm{~s}$, the simulation results are shown in Fig.7 and Tab.1.

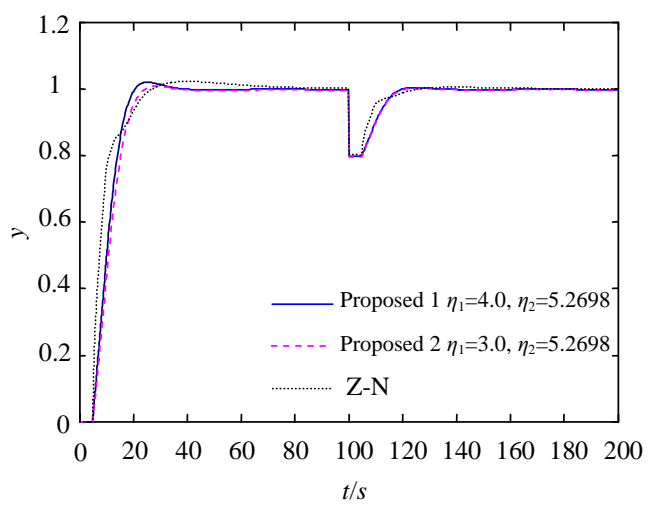

Fig.7 Step responses with nominal process for example 2

To confirm the robust performance of the proposed method, if the process model generates perturbation Eq.(52), the simulation results for the model mismatch with Z-N methods are given in Fig. 8 and Tab.1

$$
G_{2}^{\prime \prime}(s)=\frac{1.1}{9.6 s^{1.5}+6 s^{0.75}+1} e^{-4.32 s}
$$

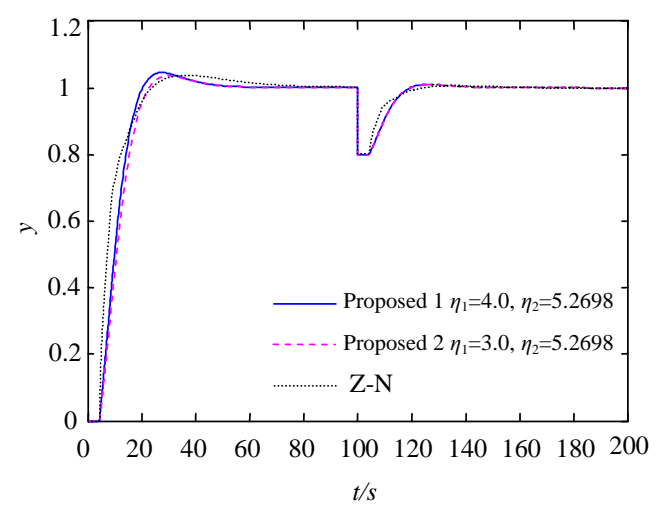

Fig.8 Step responses with perturbed process for example 2

It can be seen from Fig. 7 and 8 that both the 2-DOF FO-PID controller and the traditional PID controller 
designed following the proposed method are effective. Fig. 7 and 8 show step response of the proposed method which is compared with first $\mathrm{Z}-\mathrm{N}$ tuning rule for example $G_{2}$, the simulation results show the 2-DOF FO-PID controller can achieve higher performance than the traditional PID controller from Tab.1. The proposed method shows significant advantages both in set-point tracking and disturbance rejection. In addition, the proposed method has smaller values of overshoot and $J_{\text {ITAE }}$ when the process model $G_{2}$ generates perturbation.

Example 3. A class of integrating fractional order $(0<\alpha<2)$ process with dead-time [43] considered has the following transfer function

$$
G_{3}(s)=\frac{K}{s\left(T s^{\alpha}+1\right)} e^{-L s}=\frac{1}{s\left(0.6 s^{1.5}+1\right)} e^{-0.1 s}
$$

In [43] the achievable region of tuning a FO-[PD] $]^{\mu}$ controller in the gain crossover frequency phase margin plane was obtained. Also, the continuity of this region and uniqueness of the tuned parameters are investigated, and the necessary and sufficient conditions for applicability of this tuning method were derived.

According to the design scheme in this paper, by approximating the dead-time term of the process model with the first-order Taylor series, the 2-DOF FO-PD ${ }^{\mu}$ controller can be obtained based on the proposed design method as the following form:

$$
C_{3}(s)=\frac{1}{\left(\eta_{2}+0.1\right)}\left(1+0.6 s^{1.5}\right)
$$

When $M_{\mathrm{s}}=1.7$ is chosen, the adjustable parameter $\eta_{2}=0.0704$ can be obtained via Eq.(41), meanwhile adjustable parameter $\eta_{1}=0.06$ and $\eta_{1}=0.04$ are selected respectively. In [43] the type of FO-[PD] ${ }^{\mu}$ controller is given as follows:

$$
C(s)=6.4699(1+0.4252 s)^{1.8042}
$$

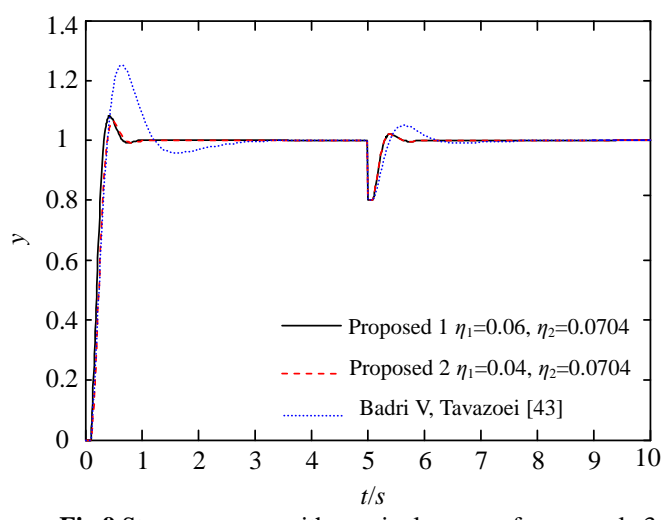

Fig.9 Step responses with nominal process for example 3

Considered that the model is accurate, the closed-loop system is simulated with a unit step change at $t=0$ and a step disturbance with value of 0.2 at $t=5 \mathrm{~s}$, the simulation results are shown in Fig.9 and Tab.1.

Now suppose that the process model generates perturbation Eq.(56). The simulation results for the model mismatch for the methods in [43] are given in Fig.10 and Tab.1.

$$
G_{3}^{\prime \prime}(s)=\frac{1.2}{s\left(0.54 s^{1.5}+1\right)} e^{-0.12 s}
$$

It can also be seen clearly that, the response speed of closed-loop system [43] is slow and has big overshoot for example $G_{3}$ from Fig.9 and Tab.1, the process goes into steady state after oscillation. Also, performance indicators of overshoot and $J_{\mathrm{ITAE}}$ remain smaller values under model parameters variations when the designed controller is applied in Tab.1 and Fig.10, which means the system with designed 2-DOF FO-PID controllers has stronger robustness against model parameters changes than $\mathrm{FO}-[\mathrm{PD}]^{\mu}$ controller in [43]. Comparing with the FO-[PD] ${ }^{\mu}$ Eq.(55) controller, the designed 2-DOF FO-PID controller is easier to implement and tune on-line, so it is more convenient in engineering. Additionally, we also demonstrate the better performance of the proposed method by smaller values of indices in Tab. 1 .

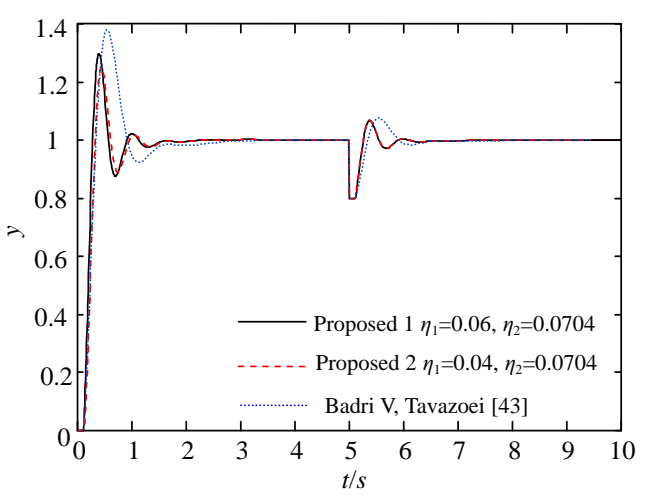

Fig.10 Step responses with perturbed process for example 3

Fig.5-10 show comparisons of the proposed method with other methods such as Z-N and IMC [16], and we show global stability region of the parameters for FO-PID controller [43]. In the above simulation study, when $\eta_{1}$ increases, dynamic response speed of the closed-loop system becomes faster, these results of three different types of processes with performance indices are listed in Tab.1.

\section{Conclusion}

In this paper, we propose a simple design approach of 2-DOF FO-PID controller based on IMC for FO processes with dead-time. The robustness and dynamic performance of the closed-loop control system can be decoupled from each other, the shortcoming of the conventional IMC control could be solved, and then performance of set-point tracking and robustness can be adjusted independently and monotonously. It is found that 2-DOF control scheme retains the property of a conventional IMC, thus can achieve good robust performance if tuned properly. The adjustable parameter $\eta_{2}$ of 2-DOF FO controller is directly related to the robustness of the closed-loop system based on maximum sensitivity $M_{s}$, and the analytical expression is given between $M_{s}$ and parameter $\eta_{2}$. According to the dynamic performance requirement of practical system, the parameters $\eta_{1}$ can also be selected easily. Additionally, dynamic response speed of the closed-loop system becomes faster when parameter $\eta_{1}$ increases. The dead-time term of process model is approximated by a first-order Padé or Taylor approximation, and then the different types of 2-DOF FO-PID controllers can be obtained for three classes of FO processes with dead-time respectively. The simulation results show that 
the proposed method is not only simple in design and convenient parameter tuning, but also provide a better dynamic performance of both set-point tracking and a better robustness against the parameters perturbation of the system.

Tab 1 Performance indices for example 1-3

\begin{tabular}{cccccc}
\hline \multirow{2}{*}{ Process } & Method & \multicolumn{2}{c}{ Nominal case } & \multicolumn{2}{c}{ Mismatch case } \\
\cline { 2 - 6 } & & $\sigma / \%$ & $\boldsymbol{J}_{\mathrm{ITAE}}$ & $\sigma / \%$ & $\boldsymbol{J}_{\text {ITAE }}$ \\
\hline \multirow{3}{*}{ Example 1 } & Proposed 1 & 0 & 2.9957 & 1.47 & 3.9316 \\
& Proposed 2 & 0.24 & 2.9152 & 1.12 & 3.8884 \\
& Z-N & 8.18 & 7.3186 & 8.89 & 7.7436 \\
\cline { 2 - 6 } Example 2 & IMC & 4.76 & 12.6470 & 4.65 & 10.7773 \\
& Proposed 1 & 2.17 & 294.3054 & 4.56 & 338.3351 \\
& Proposed 2 & 0.54 & 298.007 & 3.65 & 341.7926 \\
\cline { 2 - 6 } Example 3 & Z-N & 2.41 & 325.5919 & 3.92 & 348.2176 \\
& Proposed 1 & 2.12 & 0.2468 & 25.22 & 0.4171 \\
& Proposed 2 & 2.15 & 0.2520 & 29.88 & 0.4155 \\
\hline
\end{tabular}

\section{Acknowledgements}

The authors would like to thank the financial support provided by the National Natural Science Foundation of China (61290323, 61333007, 61473064), the National Science Foundation of Shanxi Province of China (No: 2012011027-4, 2011011011-2) and Research Project Supported by Scholarship Council of China (No: 2013-092).

\section{References}

[1] Caponetto R. Fractional order systems: modeling and control applications. World Scientific; 2010.

[2] Monje CA, Chen YQ, Vinagre BM, Xue DY, Feliu V. Fractional-order systems and controls: fundamentals and applications. Springer-Verlag; 2010.

[3] Gabano JD, Poinot T. Fractional modelling and identification of thermal systems. Signal Processing 2011; 91(3): 531-541.

[4] Petras I, D Sierociuk, Podlubny I. Identification of parameters of a half-order system. IEEE Trans Signal Pro 2011; 60(10): 5561-5566.

[5] Das S, Gupta A. Fractional order modeling of a PHWR under step-back condition and control of its global power with a robust controller. IEEE Trans Nuclear Sci 2011; 58(5): 2431-2441.

[6] Zhao CN, Xue DY, Chen YQ. A fractional order PID tuning algorithm for a class of fractional order plants. In: Proceedings of the IEEE International Conference on Mechatronics and Automation. Niagara Falls, Canada; 2005.p.216-221.

[7] Sierociuk D, Dzieliński A, Sarwas G, Podlubny I, et al. Modelling heat transfer in heterogeneous media using fractional calculus. Phi Trans of the Royal Society A: Mathematical, Phy Eng Sci 2013; 371(1990).

[8] Malek H, Luo Y, Chen YQ. Identification and tuning fractional order proportional integral controllers for time delayed systems with a fractional pole. Mechatronics 2013; 23(7):746-754.

[9] Petráš I. Fractional-order memristor-based Chua's circuit. IEEE Trans Circuits Syst II, Exp Briefs 2010; 57(12): 975-979.

[10] Nataraj P SV, Kalla R. Computation of spectral sets for uncertain linear fractional-order systems. Communications in Nonlinear Science and Numerical Simulation 2010; 15(4): 946-955.

[11] Feliu BV, Pérez RR, Garcia FJC, et al. Smith predictor based robust fractional order control: Application to water distribution in a main irrigation canal pool. J Process Control 2009; 19(3): 506-519.

[12] Podlubny I. Fractional-order systems and $\mathrm{PI}^{\lambda} \mathrm{D}^{\mu}$ controller. IEEE Trans Autom Control 1999; 44(1): 208-214.

[13] Luo Y, Chen YQ. Fractional order [proportional derivative] controller for a class of fractional order systems. Automatica 2009; 45(10): 2446-2450.

[14] Li HS, Luo Y, Chen YQ. Fractional-order proportional and derivative
(FOPD) motion controller: tuning rule and experiments. IEEE Trans Control Syst Technol 2010; 18(2): 516-520.

[15] Luo Y, Chen YQ. Stabilizing and robust fractional order PI controller synthesis for first order plus time delay systems. Automatica 2012;8(9): 2159-2167.

[16] Valério D, Da Costa JS. Tuning of fractional PID controllers with Ziegler-Nichols-type rules. Signal Processing 2006; 86(10): 2771-2784

[17] Hamamci SE. Stabilization using fractional-order PI and PID controllers. Nonlinear Dyn 2008; 51(1-2): 329-343.

[18] Gao Z, Yan M, Wei JX. Robust stabilizing regions of fractional-order $\mathrm{PD}^{\mu}$ controllers of time-delay fractional-order systems. J Process Control 2014; 24(1): 37-47.

[19] Merrikh. Bayat. F, M Karimi-Ghartemani. Method for designing $\mathrm{PI}^{\lambda} \mathrm{D}^{\mu}$ stabilisers for minimum-phase fractional order systems. IET Control Theory Appl 2010; 4(1): 61-70.

[20] Hamamci SE. An algorithm for stabilization of fractional-order time delay systems using fractional-order PID controllers. IEEE Trans Autom Control 2007; 52(10): 1964-1969.

[21] Wang DJ, Gao XL. $H_{\infty}$ design with fractional-order PD ${ }^{\mu}$ controllers. Automatica 2012; 48(5): 974-977.

[22] Wang DJ, Li Wei, Guo ML. Tuning of $\mathrm{PI}^{\lambda} \mathrm{D}^{\mu}$ controllers based on sensitivity constraint. J Process Control 2013; 23(6): 861-867.

[23] Das S, Saha S, Das S, Gupta A. Improved model reduction and tuning of fractional-order $\mathrm{PI}^{\lambda} \mathrm{D}^{\mu}$ controllers for analytical rule extraction with genetic programming. ISA Trans 2012; 51(2): 237-261.

[24] Cheon Yu Jin, Kyung Hwan Ryu, Su Whan Sung, Jietae Lee, In-Beum Lee. PID auto-tuning using new model reduction method and explicit PID tuning rule for a fractional order plus time delay model. J Process Control 2014; 24(1): 113-128

[25] Das S, Saha S, Das S, Gupta A. On the selection of tuning methodology of FOPID controllers for the control of higher order processes. ISA Trans 2011; 50(3): 376-388.

[26] Padula F, Visioli A. Tuning rules for optimal PID and fractional-order PID controllers. J Process Control 2011; 21(1): 69-81.

[27] Das S, Pan I, Das S. Performance comparison of optimal fractional order hybrid fuzzy PID controllers for handling oscillatory fractional order processes with dead time. ISA Trans 2013; 52(4): 550-566.

[28] Das S, Saha S, Das S, Gupta A. A novel fractional order fuzzy PID controller and its optimal time domain tuning based on integral performance indices. Eng Appl of Artificial Intelligence 2012; 25(2): 430-442.

[29] Tavakoli KM, Haeri M. Fractional order model reduction approach based on retention of the dominant dynamics: Application in IMC based tuning of FOPI and FOPID controllers. ISA Trans 2011; 50(3): 432-442.

[30] Vinopraba T, Sivakumaran N, Narayanan S, Radhakrishna TK. Design of internal model control based fractional order PID controller. J Control Theory Appl 2012; 10(3): 297-302.

[31] Bettayeb M, Mansouri R. Fractional IMC-PID-filter controllers design for non-integer order systems. J Process Control 2014; 24(4): 261-271. 
[32] Oustaloup A, Sabatier J, Lanusse P. From fractal robustness to CRONE control. Fractional Calculus Applied Analysis 1999; 2(1): 1-30.

[33] Xue DY, Chen YQ. A comparative introduction of four fractional order controllers. In: Proceedings of the 4 th world congress on intelligent control and automation. Shanghai, China; 2002.p.3228-3235.

[34] Monje CA, Vinagre BM, Feliu V, Chen YQ. Tuning and auto-tuning of fractional order controllers for industry applications. Control Eng Pract 2008; 16(7): 798-812.

[35] Bettayeb M, Mansouri R. IMC-PID-fractional-order-filter controllers design for integer order systems. ISA Trans 2014; 53(5): 1620-1628.

[36] Ahn H S, Bhambhani V, Chen YQ. Fractional-order integral and derivative controller for temperature profile tracking. Sadhana 2009; 34(5): 833-850.

[37] Debbarma S, Saikia LC, Sinha N. Solution to automatic generation control problem using firefly algorithm optimized $\mathrm{I}^{\lambda} \mathrm{D}^{\mu}$ controller. ISA Trans 2014; 53(2): 358-366.

[38] Bruzzone, Luca, Pietro F. Fractional-order control of a micrometric linear axis. J Control Sci Eng 2013; 2013: 4.

[39] Astrom KJ, Panagoulos H, Hagglund T. Design of PI controllers based on non-convex optimization. Automatica 1998; 34(5): 585-601.

[40] Zhao ZC, Liu ZY, Zhang JG. IMC-PID tuning method based on sensitiveity specification for process with time-delay. J Cent South Univ Technol 2011; 18(4): 1153-1160.

[41] Wang YG, Cai WJ. Advanced proportional-integral-derivateive tuning for integrating and unstable processes with gain and phase margin specifications. Ind Eng Chem Res 2002; 41(12): 2910-2914.

[42] Narang A, Shah SL, Chen T. Continuous-time model identification of fractional-order models with time delays. IET Control Theory Appl 2011; 5(7): 900-912.

[43] Badri V, Tavazoei MS. On tuning fractional order [proportional-derivat -ive] controllers for a class of fractional order systems. Automatica 2013; 49(7): 2297-2301. 